\title{
On incorporating physical building parameters into neural net models of dynamic thermal behaviour
}

\author{
N. ROBINSON*, S. BUREK*, G. BURNS\# \\ *Department of Energy and Environmental Technology, Glasgow \\ Caledonian University, \\ Cowcaddens Road, Glasgow, G4 OBA, Scotland. \\ e-mailnro@uk.ac.gcal \\ \#Department of Engineering, Glasgow Caledonian University, \\ Cowcaddens Road, Glasgow, G4 OBA, Scotland.
}

\begin{abstract}
This paper investigates the ability of feedforward artificial neural networks to distinguish between data-sets from a dynamic model of the thermal behaviour of buildings with different structural and operational parameters. Results are presented of preliminary attempts to incorporate both physical building parameters and dynamic data into a general model. Once developed, this technique should enable use of data-based ANN models as mechanistic investigative tools.
\end{abstract}

\section{Keywords}

Neural networks, building, thermal behaviour, modelling

\section{INTRODUCTION}

\subsection{Context}

Fully-connected feed forward neural networks are well suited to modelling complex or only partially understood systems for several reasons - for example: due to bounded transfer functions, they can mimic highly non-linear sequences (Montague and Morris, 1994) and they are capable of adopting a wide variety of mathematical structures due to their ability to emphasise or ignore inputs. The ability to predict time-series data in the absence of a mechanistic understanding of the system is clearly useful for practical control purposes. Through learning from examples, an artificial neural network (ANN) stores information, describing the system, in its weights and biases. 
Models of building thermal behaviour can be used to design energy-efficient buildings or assess the likely benefits of energy-saving measures. Many such models exist (Clark and Irving, 1988) employing a wide variety of modelling techniques featuring analogues of what the modeller considers to be the dominant physical mechanisms at work. An artificial neural network model requires fewer assumptions regarding its mathematical structure. However, it can only be expected to accurately predict the behaviour of the building which produced its training data.

The authors are attempting to develop a method of extracting meaningful physical building information from data-based ANN models of dynamic building behaviour by training a general model on a mixture of dynamic and pre-determined parameters defining the structure of the building and its mode of operation. Once developed, such a technique could be very easily translated into other areas of environmental or policy-making software design for use in areas where there is a database but limited understanding and in which it may be helpful to be able to make predictions concerning the outcome of a set of actions or policies.

Before using data from real buildings, it was decided to develop the technique using computer-generated data. A computer model of a greenhouse was used to generate several sets of hourly temperatures and auxiliary heat inputs as training data for different greenhouse structures and modes of operation.

\subsection{Rationale}

ANN models trained to be specific to each data-set were considered useful in establishing the nature of the data to be used in this study. For the ANN models to be considered specific to the data on which they were trained, they should yield significantly higher prediction errors when applied to the other data-sets. In the case that they are shown to be specific in this way, they must contain information representing the relative values of the greenhouse parameters used in generating the data-sets.

The degree to which each model is specific to its training data may be considered to be a measure of the relative importance of the parameter set (structural and operational information) which distinguish it from the other greenhouses.

A general model, capable of predicting the heat input, $Q$, to the same accuracy as a specific model, would need to alter its mode of operation according to the structural and/or operational greenhouse parameters used to generate the data, Therefore, it should need access to this information as additional inputs. Such a general model would allow the investigation of the links between a building's architecture and its thermal performance.

\section{METHOD}

Each data set was modelled using its own ANN model. All were feedforward neural networks with sigmoidal transfer functions in the hidden layer neurons and linear transfer functions in the output neurons. The ANNs were trained using Jacobs' (1988) delta-bar-delta back-propagation algorithm with a momentum term. Feedforward neural networks were chosen in preference to recurrent neural networks because although recurrent neural networks, are well-suited to modelling dynamic behaviour (Si and Pang, 1993), they are inherently more difficult to dissect. A feedforward neural network can be used to model dynamic time-series by carrying out static mappings using past outputs as inputs in addition to the suspected system inputs. 
The ANNs were trained and analysed on a PC and coded using C. For an introduction to artificial neural networks, see, for example Haykin (1994).

\subsection{Creation of specific ANN models}

A complex simulation of the thermal behaviour of greenhouses, based on fundamental heat transfer principles, was used to generate hourly data for the heating and internal temperature, given ambient weather data. Twenty-five sets of hourly data were generated, each set representing the behaviour of a greenhouse with slightly different physical or operating parameters to the rest. Each set contained one year of hourly internal temperature and heat input information along with weather information.

All the data was scaled from minus one to plus one. The weather data was common to all the data sets but the range of the heating input $(\mathrm{Q})$ and the internal temperature $(T)$ varied from one data set to another. In order for it to be possible for all the data sets to be modelled by one general model, a common scaling had to be used for all data sets. In order to ensure that data sets with large ranges did not dominate the modelling process and the subsequent analysis, the RMS error derived from attempting to map samples from any one data set is reported as a percentage of the range of $Q$ for that data set.

Of the 8760 sequential input-output pairs in each data set, 1600 pairs were selected at random to be used as a training set and the rest as a validation set. The training sets were divided in half. 800 samples were used to adjust the weights and biases of the network. The other 800 pairs were used as a sample test set, to gauge to what extent the model would generalise to data which had no direct influence on its training.

Twenty-five ANN models (one for each data set) were trained, using back propagation, to predict a value for the heat input (Q) at time $t$, based on the values of $Q$ and $T$ (the internal temperature) at time $\mathrm{t}-1$ hours and the weather data (solar radiation and ambient temperature) at time t.

Each of the twenty-five ANN models consisted of six neurons in the input layer - four for the hourly weather data and one for each of the delayed outputs $(\mathrm{Q}(\mathrm{t}-1)$ and $\mathrm{T}(\mathrm{t}-1))$. There were two neurons in one hidden layer and one neuron as the output $Q(t)$. The number of neurons in the hidden layer was chosen using an ANN feature detector as described by Montague and Morris (1994).

The criterion for the training was the output, $Q(t)$ from the $A N N$, compared with the value for $Q(t)$ generated by the mechanistic computer simulation and the difference termed the error. The set of weights which yielded the smallest root-mean-squared (RMS) error when applied to the sample test set was saved and training continued until no significant improvements were being made. Each ANN model was then validated using its own validation set and found to produce a similar RMS error to that obtained on the sample test set. The extent to which these models were specific to their own data was explored by running each model with every validation set and examining their RMS errors (figure 1).

\subsection{Creation of general model}

A general ANN model was trained using all twenty-five sets of training data. In addition to the six input fields mentioned above, another seven were added to allow input of parameter values. It was anticipated that this model would use the parameter data to make appropriate predictions. 
To act as a control, another ANN model was trained on all twenty-five sets of training data but was not given access to parameter data.

The general model and the control were deliberately over-specified - both ANN models contained two hidden layers with thirteen neurons in the first and ten in the second. Overfitting was avoided by using a sample test set and saving the best nets as they arose.

\subsection{Summary of terms}

- Specific models are each trained using weather data, internal temperature and heating data only. That is to say that there is no explicit input of structural or operating parameters though these are implied by the dynamic data.

- Intended data set refers to the validation data set that a specific ANN model was trained to be able to map.

- The general model is trained with the weather, internal temperature and heating data used to train all twenty-five specific models plus the structural and operating parameters used to create all twenty-five data sets.

- The control model is trained in the same way as the general model but is not given the structural and operating parameters as inputs.

- Using a standard shorthand, the specific ANN models had a 6-2-1 architecture ( 6 inputs, 2 hidden layer neurons, 1 output) whilst the general model can be described as 13-13-10-1 and the control model as having a 6-13-10-1 architecture (i.e. the control model is missing the seven parameter data inputs).

\section{RESULTS AND DISCUSSION}

All twenty-five models were successfully trained to $3 \pm 0.75 \%$. The results of running each model with each validation data-set are shown in figure 1. Figure 1 shows a steep sided valley running diagonally through the matrix of prediction errors. The points on this diagonal represent those runs in which the specific models were applied to their appropriate validation sets. The predominance of relatively high errors to both sides of this diagonal indicate there is a significant degree of specificity to the models. The presence of low error points away from this diagonal indicates that several models with different operating or structural parameters can share similar modes of behaviour. 


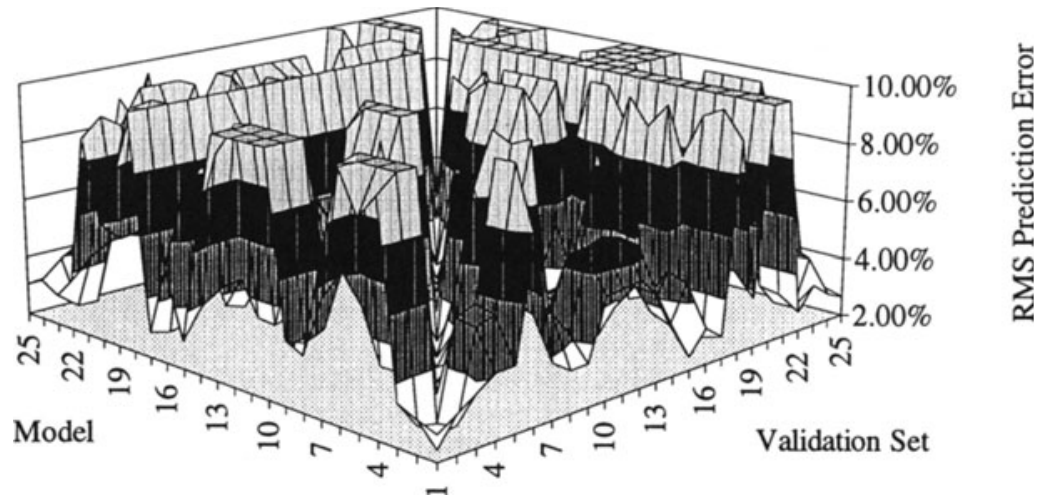

Figure 1 prediction error produced by running all models with all data sets.

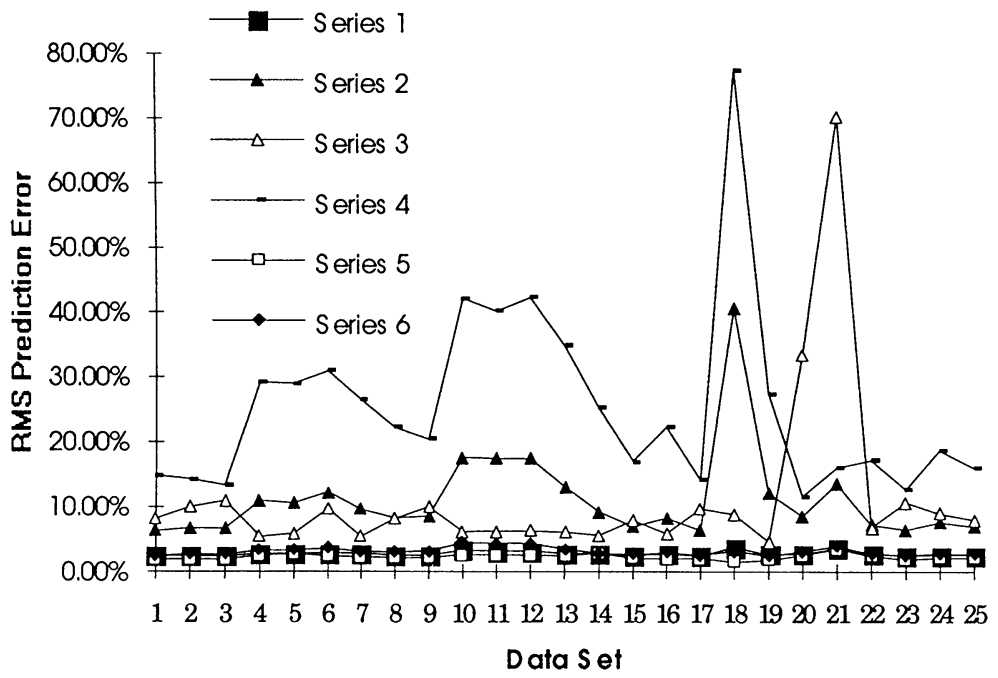

Figure 2a RMS prediction error plotted against data-set. Series:

1. Shows prediction error against validation set for the specific models run on their intended data sets.

2. Shows the average RMS error across all twenty-four ANN models when applied to each validation set.

3. Gives an error value for each model when tested across all validation data sets (in this case the $\mathrm{x}$-axis represents the twenty-five specific models rather than their validation data sets). 
4. Shows the performance of rather poor kernel model. For every sample, the kernel chooses one model at random from the twenty-five specific models. The kernel is not given the parameter data which might enable it to choose an appropriate model.

5. Shows the performance of the general model (see figure $2 b$, series 2 , for more detail).

6. Shows the performance of the control model (see figure $2 b$, series 3 , for more detail).

\subsection{Interpretation and analysis}

As can be seen from figure $2 \mathrm{a}$, the performance of the general model at least matched that achieved by the specific models when tested on their own validation sets. This would seem to imply that the general model is able to use the parameter data in order to make a prediction appropriate to the particular data-set being used. Unfortunately though, the control also performs very well compared to the results obtained from random application of the twentyfive specific models to the problem of mapping any particular sample (i.e. figure 2a, series 4). Figure 2a series 2 and series 3 illustrate the extent to which the specific models are specific to their own data.

Examination of figure 2a, series 3, reveals that models 20 and 21 yield distinctly high average RMS error values when tested across all data sets. This implies that these models are more specific to their own training data sets than the other models. Also from figure 2a, series 2, data set 18 yields a high average RMS error value when used in testing across all models. This implies that data set 18 contains data describing a distinctly different mode of behaviour (due to a significantly different set of operating parameters). Data sets 18,20 and 21 were produced using parameter sets in which the set-point temperature of the greenhouse ( i.e. the temperature below which the heating system operates) was varied as in table 1.

Table 1

\begin{tabular}{lccc}
\hline Model & Set-point ${ }^{\circ} \mathrm{C}$ & $\begin{array}{r}\text { Total Yearly Q } \\
\left(\mathrm{KJ} / \mathrm{m}^{2}\right)\end{array}$ & $\begin{array}{c}\text { Maximum hourly Q } \\
\left(\mathrm{KJ} / \mathrm{m}^{2}\right)\end{array}$ \\
\hline 18 & 0 & 49734 & 562 \\
19 & 5 & 729306 & 1350 \\
20 & 15 & 6166469 & 2704 \\
21 & 20 & 10594300 & 2704 \\
\hline
\end{tabular}

From a mechanistic point of view, one would expect large variations in set-point temperature to produce significant changes in the dynamic behaviour of the system as well as the total yearly energy used (i.e. at an average outdoor temperature of $11.25^{\circ} \mathrm{C}$, greenhouses 18 and 19 would experience a net gain in heat energy over the year whereas greenhouses 20 and 21 would experience the opposite). 


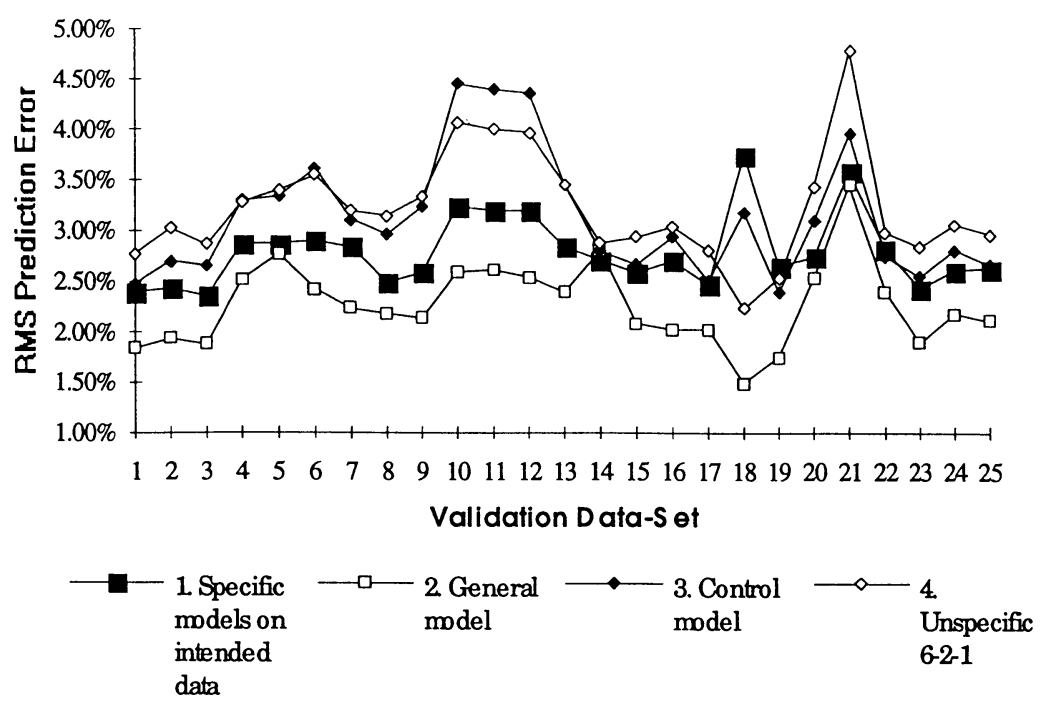

Figure 2b RMS prediction error plotted against validation data set

Figure $2 \mathrm{~b}$ allows more detailed inspection of the relative performances of the general and control models tested across all validation data sets; the specific models when tested on their intended validation data sets; an unspecific 6-2-1 trained and tested across all data sets. From figure $2 \mathrm{~b}$, it can be seen that, in most cases, the control model performed less well than the specific models and that the general model performed somewhat better.

In the case of data set 18 , the specific model performed worst of all. Repeated training of model 18 yielded no improvements even though the training and sample test set errors could be could be as little as half of that found when testing on the validation set. Further attempts using a 6-1-1 architecture yielded similar results seeming to imply that data set 18 contains 'simple' data and that the use of two or even one hidden layer neuron leads to an over-dimensioned 1 network. This is borne out by the relatively good performance of an unspecific 6-2-1 network when tested on data set 18 . It seems reasonable to suggest that in the case of the general model and that of the unspecific 6-2-1, data set 18 formed part of a larger pattern. Whereas in the case of model 18, faced with simple data and an excess of input information and processing capacity, it could not help but overlearn.

In the case of data set 21 , it can be seen that the general and specific models failed to improve significantly on the performance of the control model. This implies that although the simple architecture used by the specific ANN models proved sufficient to model the output of the mechanistic greenhouse model for almost all parameter sets, it cannot match the mechanistic model's capacity for complex behaviour. 


\subsection{A mechanistic explanation}

Figure 3 shows there is a fairly strong positive correlation between 'peak hourly heat input' and 'total yearly heat input' ( giving a Pearson correlation coefficient of 0.79 ) but that the points representing those models in which 'set-point temperature' is varied, appear as rogues ( without these points the correlation increases to 0.96). This indicates a likelihood of there being mechanistic explanations for the difficulty encountered in accurately modelling certain modes of behaviour ( for example, in models 20 and 21, the peak heat input is limited by the capacity of the heating system so that at some occasions the set-point temperature is not achieved). The fact that the modelling procedure performs badly on data which can be seen as significantly different in a mechanistic sense, is in itself promising in the search to link databased ANN models to the physical parameters of the systems responsible for their training data.

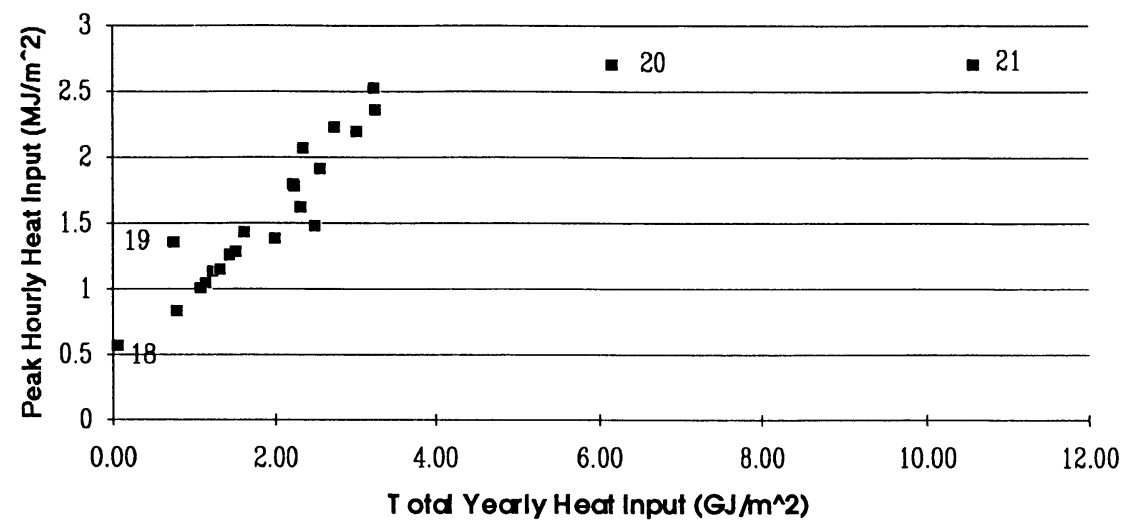

Figure 3 peak hourly heat input plotted against total yearly heat input.

ANNs with sigmoidal activation functions are recognised as 'universal approximators' ( Mitchell, 1992) (i.e. they are able to map any continuous function) and therefore a sufficiently large and complex architecture, successfully trained, should be able to reproduce the mechanistic greenhouse model in all its complexity. The 'error-backpropagation' training algorithm, being a gradient-descent method, has a tendency to yield sub-optimal solutions (Willis et al., 1991; Marshall and Harrison, 1991). This has become a problem when trying to establish whether or not a particular ANN architecture is capable of matching the dynamic behaviour produced by any/all configurations of the mechanistic model. It is for this reason that the authors are currently investigating several network training algorithms which claim to be globally convergent (Willis et al., 1991; Marshall and Harrison, 1991; Yakowitz, 1993). 


\subsection{Further tests}

In order to gauge to what extent the general model was able to make use of the parameter data, it was run with all twenty-five parameter sets in turn over all twenty-five validation data sets in turn. From this it was possible to build up a cross-testing matrix of six hundred and twenty-five RMS prediction errors, analogous to that shown in figure 1 . The result of this test was a three-dimensional surface plot which, at a glance was the same as figure 1 (and so is not included here). This was taken to imply that the general model was indeed able to make use of the parameter data as, by counter-example, inappropriate parameter data caused the general model to make erroneous predictions. Further inspection of the two cross-testing matrices (used to create the 3-d surface plots) confirmed that the general model was at least as specific in matching parameter sets to validation data sets as the twenty-five specific models were to their intended validation data sets. The ability of general models as described here, to predict the behaviour of a new system when given its parameter data, is still under investigation.

\section{CONCLUSIONS}

The specific ANN models performed generally better on their own data sets (i.e. those from which their training sets were taken) than on the other data sets. This implies two things:-

1. the data-sets used in this study described dynamic systems which were noticeably different at the resolution at which this study was carried out.

2. the individual models had been trained to a level of accuracy which made them specific to the data-set from which their training data was taken.

Therefore, this implies that the specific ANNs must have at least partial knowledge of the parameter values used in creating the data - stored in the form of the weights and biases defining each ANN model.

For a single general model to perform as well as these specific models across all twenty-five data-sets, it would need to encompass a range of modes of operation, and to switch between them depending on which particular data-set it was being asked to imitate. The general model was trained with input information connecting sets of parameters to modes of behaviour and, once trained, the general model used this parameter information to select an appropriate mode of operation.

The results presented here were obtained using data from a mechanistic, well-behaved computer simulation of the thermal performance of a relatively simple greenhouse structure. These results appear promising and the authors are in the process of developing and applying the techniques outlined here to real thermal performance data from real buildings.

\section{REFERENCES}

Montague G. and Morris J. (1994) Neural-network contributions in biotechnology, Trends in Biotechnology. Vol. 12, 312. 
Clarke J. A. and Irving A. D. - guest editors. Special Issue devoted to Building Energy Simulation. Energy and Buildings. Vol. 10 No. 3.

Jacobs R. A. (1988) Increased rates of convergence through learning rate adaptation. Neural Networks 1, 295-307.

Si J. and Pang L.(1993) Recurrent neural networks for dynamic system modelling, Proceedings of the 1993 International Symposium On Intelligent Control. 364-369.

Haykin S. (1994) Neural Networks: A Comprehensive Foundation. Macmillan, New York.

Mitchell J. (1992) Comparing feedforward neural network models for time series prediction, Neural Computing Research and Applications, Part One, 175-182.

Willis M. J.; Di Massimo C.; Montague G. A.; Tham M. T. and Morris A. J. (1991) Artificial neural networks in process engineering, IEE Proceedings D, Vol. 138 No. 3 219-266.

Marshall S. J. and Harrison R. F. (1991) Optimization and training of feedforward neural networks by genetic algorithms, Artificial Neural Networks - 2nd. International Conference, 39-43.

Yakowitz S. (1993) A globally convergent stochastic approximation, SIAM J. Control and Optimization, Vol. 31 No. 1, 30-40.

\section{BIOGRAPHY}

Nick Robinson graduated with a BSc in environmental science and independent studies from Lancaster before moving to Glasgow Caledonian for an MSc in energy systems and environmental management. $\mathrm{He}$ is currently studying for a $\mathrm{PhD}$ at Glasgow Caledonian University.

Dr. Stas. Burek graduated from Cambridge in 1979 as an engineer and has worked in the energy field for the last fifteen years. He gained a $\mathrm{PhD}$ in solar energy from Cranfield in 1987 and is currently a Senior lecturer at Glasgow Caledonian University.

Dr. George Burns graduated from the University of Strathclyde with a Bsc in physics, then a $\mathrm{PhD}$ in electronics. He is currently a senior lecturer and divisional leader in the Dept. of Engineering at Glasgow Caledonian University. 